\title{
Understanding User Needs
}

\section{Sharon McMeekin}

\section{DPC Technology Watch} Guidance Note July 2021 


\section{Introduction}

Developing a digital preservation program is a complex and challenging undertaking and there are many competing concerns. The temptation is to prioritise the issues that seem most pressing, like ingest and storage. This can reduce the time available for activities that do not seem essential, like understanding user needs. Such activities might seem like a luxury rather than a necessity.

But implementing a robust approach to understanding user needs through an analysis should be a key component of any digital preservation program, into which it is worth investing time and effort. A clear understanding of user needs can help guide how your organization approaches almost every aspect of its digital preservation work. This is particularly true in the case of access provision, where understanding your current and potential users is essential. Understanding what our users want and need provides us with the end goal for any digital preservation program.

\section{Why it is Important to Understand Your Users' Needs}

It is possible to define the ultimate aim of digital preservation as enabling continued access to digital content, and allowing users to gain value from that content. Without understanding what content users will want or need, and how to provide meaningful access to it, you will not be able to fulfil this aim. Therefore, we can not make any claims about the success of our preservation activities, without an understanding of user needs to measure them against.

The fundamental role that user needs analysis plays in developing digital preservation capabilities can be further reinforced by examining how many areas of decision-making it can influence. At a broad level across the digital preservation lifecycle this can include:

- Informing the selection of content for preservation.

- Helping to advocate for resources by demonstrating demand or need.

- Aiding in the identification of the significant properties of content that need to be preserved.

- Informing the creation of digital preservation policy and procedures.

- Understanding the metadata and documentation that will be needed.

- Influencing preservation planning, identifying methods that will produce reusable content.

As mentioned above, understanding user needs is particularly important when developing access provisions. It can provide guidance so that we will be able to:

- Understand accessibility requirements.

- Identify tools to aid reuse of content.

- Know which formats users will be able to access.

- Know what licences will be required to enable reuse.

- Understand how to present content to make it discoverable.

- Know what guidance users will need to open and use the content.

Once you have decided to incorporate user needs analysis into your digital preservation programme development, creating a well-considered and clearly structured plan will help you move forward.

\section{Developing a Plan for User Needs Analysis}

Developing a robust plan is the first step towards carrying out a successful user needs analysis exercise. It is important not to just jump into the process, but to carefully consider what you want to achieve and how best to reach those aims. Your plan should contain the standard elements of a project plan such as key aims, tasks, a timeline, risks, responsibilities, and potential benefits. You will 
also need to include sections specific to user needs analysis, such as user groups you will target, how you will communicate with them, data capture methods, and how you will report and utilize results.

It is important to remember that users may change over time, and through good access provision and engagement, we may attract new user groups. You should plan to repeat analysis at regular intervals and to consider users you do not currently reach. Designing a robust approach that can be repeated will help you capture data you can compare over time. So, what should we think about before beginning a user needs analysis exercise? To start it is useful to consider questions such as:

- What resources are available to you?

- Do you have colleagues who have experience of completing user needs analysis?

- Does your organization have subscriptions to tools that could help with the process?

- What data does your organisation already collect about users that can help?

- What are the key questions that you need answers to?

- Is there a particular issue you want feedback on or a tool you would like to test?

- Do you need rich data about how users interact with your site? Or simple information like the browsers or devices they use?

- Do you want to know about the types of digital content your users are interested in?

- Who are your key user groups and what methods of analysis are they likely to respond to?

- Do you have a local user base that could attend focus groups in person?

- Would an online survey be more likely to gain engagement?

- Would asking users to complete a practical task produce more useful feedback?

\subsection{Defining User Groups}

It is particularly important when planning your user needs analysis to consider how you will define your user groups. Will you only have one clearly defined group? Or will you have multiple groups with different needs? Are the groups large or small? Are they dispersed or local? Do these user groups already interact with your organization, or will you be hoping to attract new user groups?

If you will have multiple user groups, you should decide how to define the groups in a way that is both representative of them, as well as useful to you. Groupings could include how they might use the content, the types of content they are interested in, or the skills they might be expected to have. If you have multiple, diverse user groups, you may need to employ different methodologies when gathering information, selecting the methodologies most likely to engage each group.

Some types of user groups you might consider include:

- By discipline, e.g. researchers in social science or historians.

- By sector, e.g. journalists or public servants.

- How the resources will be used, e.g. by artists, data scientists, or genealogists.

- By a particular demographic, e.g. by age, location, or language.

- By accessibility requirements, e.g. those requiring assistive technologies like screen readers.

- Or even as broad as the general public.

Choosing a user group as broad as the general public should be considered with caution. It can make it difficult to clearly understand how content will be used, define needs, and understand skill levels. 


\section{Methods for User Needs Analysis}

There are a variety of methods available for user needs analysis and selecting the correct method(s) is essential to ensuring that the process is successful. When selecting the method(s) you will use you should consider:

- What resources do you have available?

- Which method(s) are most likely to engage your user group(s)?

- What information you need to capture from the process?

$\circ$ Do you need high-level facts and figures and/or more detailed information?

- Will you be gathering information to influence decisions about:

- Specific issues (e.g. how to facilitate resource discovery)?

- A particular element of digital preservation (e.g. providing access or selection)?

O Or in relation to the full lifecycle?

Each method will produce different types of information and may be better or worse suited to the outcomes you require. You may decide to use just one of these methods or a combination of a few, depending on your answers to the questions above. For each method included in the following subsections there is a short description and some pros and cons of deploying that method.

\subsection{Surveys}

Surveys are perhaps the most commonly used method for gathering information on users, their experiences, and needs. A survey provides a mechanism for the collection and analysis of the opinions and/or attitudes of a group by asking them to answer a series of questions. Question types can include yes/no, multiple choice, grading options on a scale, and both short and long written answers.

The benefits of using surveys include that they:

- Can potentially reach a lot of people without too much effort.

- Can be posted on your website or links distributed by email and social media.

- Are fairly cheap and easy to put together, both free and subscription services are available.

- Produce quantitative outputs that are easy to analyse and create digestible facts and figures.

The downsides of using surveys include that:

- Large numbers of textual responses can be hard to process and find trends.

- It is difficult to capture the full user experience; effective questions often focus on specifics.

- Phrasing of questions might then lead respondents unwittingly in a particular direction.

- You are reliant on those who have the time and inclination to fill in the survey.

\subsection{Web Analytics}

Another largely quantitative option is to use web analytics services to collect data on the use of your existing online resources. These services will automatically collect data about users to identify trends such as types of devices or browsers used, most visited pages, length of visit, etc. The most commonly used web analytics service is Google Analytics but many others are available. They are usually deployed by embedding a link to the service in relevant web pages.

The benefits of using web analytics include that:

- Once set up, data is collected automatically.

- Data will be captured on most users who visit your online services. 
- It can show patterns of usage across a broad range of user groups.

- It can capture search terms used.

- The data is easy to analyze, and services will include tools to help filter and organize data.

The downsides of using web analytics include that:

- No qualitive responses are captured, only broad patterns.

- It does not capture the user experience, only activity stats.

- It is relatively easy for users to block the capture of analytics information.

Another related source of information on user experience is the feedback received from users via forms on your website or email. While the comments, complaints, and suggestions received will likely get individual attention, it is rare that an organization will carry out a broader analysis of the issues they raise. Such an analysis can provide useful, and potentially detailed, feedback that can influence digital preservation activities.

\subsection{Focus Groups and Workshops}

A third commonly used method of analysing user needs is organising focus groups and workshops. This allows you to bring together small groups of users to describe and discuss their experiences and needs in person. Focus groups will ask participants to provide feedback on existing resources or will raise issues and potential options for discussion. A workshop will add a practical exercise to this format, which participants will be asked to provide feedback on.

The benefits of using focus groups and workshops include that they:

- Provide an opportunity for direct interaction with users and deep dive into important issues.

- Can capture multiple perspectives at once.

- Can allow you to follow-up interesting discussions as they occur.

- Allow you to generate and discuss new ideas and gain immediate feedback.

The downsides of using focus groups and workshops include that they:

- Can be difficult and expensive to organise, e.g. for disparate user groups.

- Are limited to users willing and able to attend.

- Can get off track or produce limited useful information without a skilled facilitator.

\subsection{Interviews}

Interviews are another popular method for capturing information on user needs and experiences, especially with modern communications allowing them to be conducted remotely. The interviewer will usually pose a series of structured or semi-structured questions for the interviewee, and they can range from a quite formal experience to a more casual chat.

The benefits of using interviews include that they:

- Provide an opportunity for direct interaction with users.

- Offer the ability to deep dive into important issues.

- Allow you to ask follow-on questions about answers that have raised interesting issues.

- Can be tailored to individual users to capture the most useful information.

The downsides of using interviews include that they: 
- May only reach a limited number of users without a significant investment of time and effort.

- Can be difficult to organise, especially identifying the right users to interview.

- Generate results that can be time consuming to process and analysis results.

- Can be intimidating for participants, which might lead to insincere or limited answers.

\subsection{Usability Testing}

Usability testing, or task analysis, is a technique for testing a product with users. This is often achieved by asking them to perform a set of tasks before answering questions on how they completed the tasks, how they felt about the experience, and what feedback they have. It can be used with prototype systems or with existing systems to influence how they could be improved.

The benefits of usability testing include that it can:

- Offer feedback on functionality. Is it easy to use and does it meet user requirements?

- Enable direct comparison of user experiences by assigning the same task(s) to multiple users.

- Produce both useful facts and figures and more detailed information.

The downsides of usability testing include that it may be:

- Tricky to organise depending on the user groups, locations, and access to technology.

- Difficult to define useful tasks, that are not too easy or too difficult.

- The process will reach a limited number of users, and therefore may not be representative.

\section{Using the Results}

After you have developed your User Needs Analysis plan and put it into action you will need to analyse the data and report on results. The process of data analysis will depend on the method(s) used for gathering data but might include the following stages:

- Processing: formatting the data for analysis, e.g. transferring it to a spreadsheet.

- Cleaning: Fixing issues with the data, e.g. incomplete entries, duplicates, or errors.

- Exploratory analysis: using cleaned data to find trends and calculate statistics. You may need to work through a data set multiple times to find trends using methods like keyword analysis.

When it comes to reporting results of the analysis, it is worth considering preparing two versions of the report: a short summary report with headline findings for advocacy purposes, and a full report for use in the development of your digital preservation program and related access services.

Clear recommendations should be an essential part of both versions, taking the data collected to create a coherent story of the need for change. In the summary version these can be tailored to make the case for additional resources, in the full report they should focus on clear and realistic actions to improve digital preservation capabilities and access provision.

\subsection{User Stories}

A useful technique to employ during the analysis phase is the creation of "user stories". This involves the identification of particular roles (in this case a user from a particular group) and then developing the "story" of why they are interested in your collections and how they might wish to use them. This can include defining a list of interactions they may be expected to have with a system and/or identifying the digital content that they might be interested in and how they would reuse it. It 
provides a consistent and accessible structure for understanding and presenting the needs of different user groups and can aid with clearly identifying user-led requirements for digital preservation activities.

For example, you may create a user story for a journalist searching for committee papers detailing local planning decisions. The story might then contain details of:

- The digital content they would be hoping to access.

- How they would approach searching for that content, e.g. using keywords or filters.

- How they would want to browse the search results.

- How they would want to view and interact with the digital content.

- What accompanying documentation and information they would require to facilitate their reuse of the digital content.

User stories can be as complicated or as simple as you want them to be, depending on the time and resources you have available. At a minimum for each story you should aim to create a description of the user, their motivations/aims, and list of steps and the desired outcomes.

\section{Summary}

User needs analysis is a key element of successful digital preservation, and can influence decision making across the lifecycle from acquisition to access. When embarking on an analysis exercise, it is essential to carefully plan the activities that will be involved. This will include identifying the user groups you will work with, as well as deciding which methodologies to use.

After a plan is prepared and carried out, it is important to put effort into the analysis of the collected data and the preparation of reports on results. Developing user stories as part of reporting can help focus your results. A summary report is useful for advocacy purposes, while a detailed report can inform developments in your digital preservation program, and how you approach access provision.

More detailed tips on methodologies for user needs analysis are included in the Novice to KnowHow course "Providing Access to Preserved Digital Content". 


\section{Further reading}

Digital Preservation Coalition (2015). Digital Preservation Handbook - Access. Available at:

https://www.webarchive.org.uk/wayback/en/archive/20210525231345/https://www.dpconline.org Lhandbook/organisational-activities/access

Fisher, S. (2020). 11 Tips for building effective surveys. Available at:

http://web.archive.org/web/20210524192927/https://www.qualtrics.com/blog/10-tips-for-buildingeffective-surveys/

Hotjar (2021). A beginner's guide to user \& usability testing. Available at:

http://web.archive.org/web/20210621102816/https://www.hotjar.com/usability-testing/

Hughes, J. (2019). What is Web Analytics? Your 101 on Analytics and How to Get Started. Available at: http://web.archive.org/web/20200618173649/https://themeisle.com/blog/what-is-webanalytics/

Interaction Design Foundation (2017). Usability Testing. Available at:

http://web.archive.org/web/20210616144122/https://www.interaction-

design.org/literature/topics/usability-testing

Pernice, K. (2018). User Interviews: How, When, and Why to Conduct Them. Available at:

http://web.archive.org/web/20210502165353/https://www.nngroup.com/articles/user-interviews/

Question Pro (2019). Focus group research: Steps to conduct a focus group. Available at:

https://www.questionpro.com/blog/focus-group/ [accessed $25^{\text {th }}$ June 2021]

Taylor, R. (2019). How to Conduct Interviews in Qualitative Research. Available at:

https://www.rev.com/blog/how-to-conduct-interviews-in-qualitative-research [accessed 25th June 2021]

TechTarget (2021). Web analytics. Available at:

http://web.archive.org/web/20210521055243/https://searchbusinessanalytics.techtarget.com/defi nition/Web-analytics

Wikipedia (2020). Needs Analysis. Available at: https://en.wikipedia.org/wiki/Needs analysis [accessed 25th June 2021]

Wikipedia (2021). Survey (Human research). Available at:

https://en.wikipedia.org/wiki/Survey (human research) [accessed $25^{\text {th }}$ June 2021]

Wikipedia (2021). Web analytics. Available at: https://en.wikipedia.org/wiki/Web analytics [accessed 25th June 2021]

Wikipedia (2021). Usability testing. Available at: https://en.wikipedia.org/wiki/Usability testing [accessed 25th June 2021]

Wikipedia (2021). Focus Groups. Available at: https://en.wikipedia.org/wiki/Focus group [accessed 25th June 2021]

Wikipedia (2021). User Stories. Available at: https://en.wikipedia.org/wiki/User story [accessed 25th June 2021]

Wikipedia (2021). Data Analysis. Available at: https://en.wikipedia.org/wiki/Data analysis [accessed 25th June 2021] 